Boise State University

ScholarWorks

Educational Technology Faculty Publications and

Presentations

Department of Educational Technology

6-1-2009

\title{
Online Collaborative Learning in a Project-Based Learning Environment in Taiwan: A Case Study on Undergraduate Students' Perspectives
}

Ke Zhang

Wayne State University

Shiang Wuu Peng

Kun Shan University

Jui-long Hung

Boise State University 


\title{
Online Collaborative Learning in a Project-based Learning Environment in Taiwan: A Case Study on Undergraduate Students' Perspectives
}

\author{
Ke Zhang \\ Wayne State University \\ Shiang Wuu Peng \\ Kun Shan University \\ Jui-long Hung \\ Boise State University
}

\begin{abstract}
This case study investigated undergraduate students' first experience in online collaborative learning in a project-based learning (PBL) environment in Taiwan. Data were collected through interviews of 48 students, instructor's field notes, researchers' online observations, students' online discourse and group artifacts. The findings revealed interesting phenomena as results of cultural influences as well as educational system impacts. Students experienced first handed various learning benefits of PBL in the intensive six-week period, yet voiced serious concerns about the changed role of the instructor, as well as strong reservations on peer collaboration as a result of the competitive tradition in education. Obviously, online collaborative learning and PBL critically challenged some culturally-rooted traditions in Taiwan. The study generates practical insights into the applications of online collaborative learning and PBL in Taiwan's higher education as well as implications for cross-cultural implementation of online learning.
\end{abstract}


This is an author-produced, peer-reviewed version of this article. The final, definitive version of this document can be found online at Educational Media International, published by Taylor and Francis. Copyright restrictions may apply. Doi: 10.1080/09523980902933425

\section{Introduction}

With strong government initiatives on e-learning since 2002, most traditional universities in Taiwan now offer online courses (Authors, 2006). With the increasing offerings of online learning, however, very little is done to understand students' perspectives in an oriental cultural context such as Taiwan. Likewise, project-based learning (PBL) has been widely implemented in traditional K-12 classrooms (e.g., Katz, 2000; Chard, 1992) and distance higher education institutions like the Open University (e.g., Macmillan, 1975); however, PBL, as well as online collaborative learning, is relatively new in Taiwan's educational systems. Based on critical review and analysis on elearning in all of the 147 four-year colleges and universities in Taiwan, Zhang and Hung (2006) point out that it is yet to investigate how to sensitively adopt the well-received teaching and learning methods that originated in the western educational circle, such as project-based learning and online collaborative learning in Taiwan's higher education.

With the growing number of international students in online courses or programs worldwide, it is increasingly important to understand cross-cultural impacts on learning, which in return would help develop, select and implement strategies to accommodate diverse learners and respond to the changing dynamics with cross cultural phenomena. Researchers (Allen \& Boykin, 1992; Wilson, 2001) found that in a course driven by another culture, learners would experience the mismatch of contextual cues and thus face more unexpected problems and issues in the learning process. Crump (2004) explored the issues and difficulties the newly arriving international students at universities in New Zealand. In this study, international students reported that collaborative learning was challenging, especially with very different views on ways of learning and knowledge construction through collaborative efforts among peers. Robinson's study (1999) reported the difficulty of presenting non-Western concepts when teaching about both Western and Chinese educational values and characteristics, because most of the research was based on Western psychological assumptions and methods. Lee (2004) noted that well designed webbased instruction may adopt a western constructivist approach and promote learner-centered learning. However, such an approach was very different from the traditional, teacher-centered, information-based, and test-driven instructions, which are typical or even dominant in Taiwan. In a recent study, Ku and colleagues (Ku, Pan, Tsai, Tao, \& Cornell, 2004) found that Chinese instructors consistently remained the ultimate authority and major resource in teaching and learning events. In addition, Chinese students would focus mostly on their grades in norm-referenced tests, which measure their performance relatively to each other, and thus competitions are often really intense among peers.

In general, Chinese students are more used to being "taught” by an instructor rather than being active knowledge constructors. They also tend to collect information from non-verbal channels, and thus the physical contexts of the learning environment play a very important role (Chen \& Starosta, 1998). Also, teacher's social presence is more important for Chinese students than for their western counterparts (Powell \& Harville, 1990; Tu, 2001). However, non-verbal communication channels and social presence are often limited in online learning environments. Also, given the standardized-test evaluation system, most educational institutions stick to a test-driven approach to teaching and learning. Information acquisition and recall are often the major foci of the curricula, as they are mostly tested in the educational system. Therefore, Chinese students may face more challenges and difficulties in PBL or other constructivist approaches that require active participation and collaborations.

\section{Perceptions in Online Learning Environments}

Wang (2007) examined the effects of an important cultural dimension, power distance on learners' perceptions of their online learning experiences. With participants from universities in US, South Korea, and China, the study found that the American participants and Chinese students differed significantly in terms of power distance, with the Americans having the lowest power distance score and the Chinese highest power distance score. The results indicated that Chinese students typically were not comfortable approaching their instructors for help or working with unfamiliar peers. When having to work with less-familiar peers, Chinese students would rather work individually instead of in a group. So and Brush (2008)'s recent study found that perceived social presence affected individual students' motivation to engage in collaborative learning activities as well as course satisfaction in blended learning.

Thus, it is critical to build deep understandings of students' perceptions, feelings, and experiences in online learning and PBL for culturally sensitive adoption of innovation teaching and learning methods in Taiwan and similar cultural settings. This study was designed to investigate undergraduate students' online collaborative 
This is an author-produced, peer-reviewed version of this article. The final, definitive version of this document can be found online at Educational Media International, published by Taylor and Francis. Copyright restrictions may apply. Doi: 10.1080/09523980902933425

learning experiences in a project-based learning environment in Taiwan, addressing the following research questions:

1. How do undergraduate students in Taiwan perceive online collaboration? How do they feel about online collaboration?

2. How do undergraduate students in Taiwan perceive project-based learning? How do they feel about projectbased learning?

\section{Research Design and Methods}

A case study (Stake, 1995; Yin \& Campbell, 2002) was conducted, with varied data collected from interviews, online observations, instructors’ field notes, group projects, and group online discourse.

\section{Context, Tasks and Participants}

98 college freshmen in 34 learning groups at a 4-year vocational-track university in Taiwan participated in a series of online learning activities for 6 weeks in a PBL environment. Random assignment was conducted to form these small groups in size of $3(n=30)$ or $4(n=2)$. Each group was asked to investigate and report on a different, popular social phenomenon as a group project. This course was delivered online through Wisdom Master ${ }^{\mathrm{TM}}$, the most widely applied learning management system (LMS) in Taiwan's universities. All learning materials and additional resources were available in the LMS, together with a private discussion board for each group and a public one for all students. Students could also upload and share files there. Students used the private discussion board for inside group discussions and shared their periodic submissions of sub-tasks as required on the public discussion board with comments and reviews with peers in other groups.

The group projects required a wide range of knowledge and skills, including research, survey design, data collection and analysis, advanced Excel applications, report writing, presentation, and more. The social phenomena for investigation ranged from Internet use to undergraduate students' leisure life. These topics were open-ended and sufficiently challenging, requiring students to determine the specific focus, directions, approaches, methods, tools, and others. Various coaching and scaffolding efforts were provided in the learning process.

The six week period was structured into 3 phases with 3 sub-projects requiring different major tasks due in each other week. For example, by the end of week 2, groups were required to finish their data collection instrument creation and data collection to answer the research question as they would have defined earlier in the first week. Consistently, students were required to share their drafts and sub-tasks with the class in designated public online forums and to provide constructive feedback to other groups. They were also encouraged to make revisions on previously submitted materials based on peer and instructor's comments and suggestions. The instructor provided feedback on the sub-projects but only the final projects were graded, with group members receiving the same grade for the group project.

\section{Procedure}

Written informed consent was obtained after detailed written and oral explanations about the purposes and procedures of the study. Participants profile survey was conducted at the beginning of the semester, collecting basic demographic data and previous online learning experiences. All students were assigned with a series of online learning activities that contributed to a 6-week project. After the completion of the group project, semi-structured interviews (see Appendix A for the interview protocol) were conducted in a conversational style in 12 focus groups, with 4 purposefully grouped interviewees each time.

Stratified purposeful sampling was applied to select interviewees for a comprehensive representation of different types of learners. 48 of the participants were selected for interviews, based on the levels of participation (i.e., poor, average, and superior), which were determined by the following participation statistics: frequency of reading, number of postings, and frequency of attendance. In each focus group, the researchers purposefully included all different levels of participants. Group interviews were conducted because the researchers understood that Chinese students tend to be quiet and less responsive, and a focus group may not only stimulate more responses and but also allow the interviewer to observe the interactive dynamics among different types of students. A same researcher conducted all the interviews in person at the end of the semester, which lasted from 30 to 60 minutes each. All interviews were recorded with participants' consent and later transcribed in Chinese. 
This is an author-produced, peer-reviewed version of this article. The final, definitive version of this document can be found online at Educational Media International, published by Taylor and Francis. Copyright restrictions may apply. Doi: 10.1080/09523980902933425

\section{Data Analysis}

Descriptive, statistical analysis was conducted to generate participants' profile and to compare the survey results. All qualitative data were analyzed through coding, categorizing, and drawing themes, and then triangulated with the statistical data regarding participation. All researchers are native Chinese speakers and fluent in English, and thus the qualitative analysis was conducted bilingually, with notes-taking in either or both languages, and cross-checked in both languages by at least two researchers to ensure trustworthiness. To ensure non-biased coding, the interview data were transcribed and analyzed without the participants' names, project grades, or level of participations attached to their interview responses.

\section{Findings}

Participants Profile

88 students (69 female, 19 male) completed the profile survey. They reported an average of 2.17 hours of collaborative learning experience prior to this study. 22 (25\%) students had taken completely online courses before, with eight $(9.10 \%)$ of them with one online course prior to this study and $14(15.91 \%)$ with two online courses. 13 $(14.77 \%)$ had taken courses with some online learning activities. Participants reported on average .19 hour of online collaborative learning experience prior to this study. None of the participants had any experiences with projectbased learning prior to the study.

\section{Regarding online collaborative learning:}

\section{Competition or collaboration?}

Students viewed learning as a competition and thus had strong reservations for peer collaboration. 16 out of 48 interviewees mentioned that they were upset to find out that other groups modeled their posted projects or drafts, and later developed a better one with modifications and improvements. Those who posted earlier were very upset, as they saw others had learned from them, and as a result over performed them. Although all interviewees commented that reading other groups' discussions and projects were helpful, they did not like being modeled by their peers and then eventually being over performed. So many said that they would not post their projects or share their drafts, if they had to, due to the instructor's requirement, they would wait till it's due. Clearly, these students didn't want to be over performed, and for them learning were more about competition than collaboration. However, all interviewees acknowledged that reading and reviewing other groups’ projects was very helpful, as XiangYing (female, poor participation) said, “reviewing other groups’ projects are very useful, especially [because] we can make sure that we are on the right track”. And consequently, students would often hold back on posting or sharing initial ideas or drafts in the online forums, just so that they could see what others were doing first, and wait till they were confirmed that they were on the right track. Our review of the drafts and final submissions clearly showed that many group projects were significantly improved after the peer review process, as students reviewed and revised their projects based on peer evaluations or by modeling other groups.

However, many students voiced their concerns or feeling of unfairness after being modeled and then outperformed by their peers. As ShiaoWen (Female, superior participation) said:

Say, if we post it early, somebody, we would post it on Thursday or Friday, when it was due Sunday midnight, later [we] would go in and read [others]. How strange, [other groups' posted work] was just like ours. We felt being imitated... They would model yours, imitate yours, post their own in the last minute, remove the imperfections in yours. Then you'd feel yours was not as good [as theirs], but it was yours only with some modifications... Feeling not fair, you know. It was obviously your work, your idea, your original creation, but hey they did better now...It's like the later you post [your work] the better yours would be [than others']. ...It's just unfair. They had not thought about it (the way we did), but they did [the projects] similarly as we did. But we posted it first, I went to check, and other groups just did the same thing [as we did], with only a few words changed. Just not fair.

When asked if peers sharing and reviewing were helpful in the learning process, a participant responded that: Yes, more or less. But your work would then be copied by others... Ok, you may use our work, but at least recognize it [was based on our work]... 
This is an author-produced, peer-reviewed version of this article. The final, definitive version of this document can be found online at Educational Media International, published by Taylor and Francis. Copyright restrictions may apply. Doi: 10.1080/09523980902933425

Another participant, JiaMing (male, superior participation) then commented that, "But ours was dated earlier than theirs, so the professor should know that we did it first”. Yet then another student, FeiTing (female, superior participation) voiced her concern that: "The professor may start from grading the better ones and not notice the dates at all”. More interestingly, yet another student, JingYi (female, superior participation) was concerned that, "If we then further revise ours, then our submission would be dated after the other group [who learned from our work], and it would then appear that we did ours after them...And, if we were to revise ours, we'd run out of time, next week's assignments would be due soon".

Clearly, students were very upset about being imitated and consequently being outperformed by their peers, and would like to be recognized for their original work and beneficial impacts on others' learning and performance. On the other hand, those students who had actually modeled other groups' work seemed to downplay the influence of their peers' work, as GuanYin (Famale, superior participation) acknowledged: “You mean looking at other's projects or drafts? Yes, but just a reference.” Interestingly enough, those who were modeled were upset for being outperformed yet those who had learned from their peers would not acknowledge the benefits directly. Participants were obviously in a competition, rather than collaboration mode in the learning process. They might collaborate within their own groups but certainly had more concerns and reservations about inter-group collaborations.

\section{Collaboration or cooperation?}

Interviewees shared an understanding that collaborative learning involved cooperation and chunking the task into smaller pieces for different group members to take charge of. For most of them, cooperation equaled collaboration, and was considered the only way of collaboration. Dillenbourg (1999) defined cooperation as "Partners split the work, solve sub-tasks individually and then assemble the partial results into the final output. In collaboration, partners do the work 'together.' (p. 8). Similarly, Roschelle and Teasley (1995) defined collaboration as a coordinated, synchronous activity through continuous efforts to construct and maintain shared understandings. As documented in the group online discourse and confirmed in the interviews, a typical approach to complete the group project was to discuss the task in general at the beginning, and then chunk it into smaller, individually manageable sub-tasks, and each take charge of a given number of sub-tasks. Some would also have a final assemble process as a group at the end; others, however, would have one member to put together everything at the end. Many interviewees confirmed that once the task was chunked, most students would not provide much feedback or inputs for the tasks that were assigned to other members, because they typically picked up the task that s/he felt most comfortable with, then would each focus on their own parts and would ask for help only when they could not do it alone.

\section{Regarding $P B L$}

In this PBL experience, students reported that they had gained a lot of knowledge in addition to the software program, Excel, which would be the only focus in a typical face-to-face course like this. Overall, participants reported positively about their PBL experience in this study. JiaMing (male, superior participation) mentioned that PBL had actually led to more, active, practical, and meaningful learning for him. He commented that in traditional lectures, which were a dominate practice in Taiwan and common elsewhere, he would be doing his own things, and would not be engaged in the learning process. And as a result, he would simply forget what's been lectured very soon. In PBL, they had to actively figure out what was needed to be done, and how to get there, and thus had to be consciously engaged. And when actively engaged in meaningful searches and inquiries, they learned more and better. Another participant, JiaQi (Female, superior participation) shared similar thoughts on the benefits of PBL: [In PBL, one] Can learn different things: in traditional classroom lectures, [we can] learn software usage and applications. If you do a project; however, you'll learn how to solve problems, so it changes into [ a situation in which] learning Excel is only a tiny tiny part of the process. So in PBL [one will] learn a whole lot more... like doing statistics... how to approach to [problem solving], construct [a series of] ideas, how to get other's help... Like for the content covered in the project, you'll know better now, and related knowledge, because you have to go search and find a lot of materials, so without noticing it, you'll learn a lot other knowledge.

Similarly, BaoHong (male, superior participation) shared his view:

If the teacher would keep lecturing at the podium in the front, I wouldn't stay there just listening to him, will not listen to him, but do my own things there...but if he gives you that [project], with a lot of flexibility, because you cannot just do it well without working really, really hard, so you just have to go find resources and work it out... So will learn more [in PBL], Because if you just lecture in the classroom and then assign the same practice and drill, the same stuff, you know you can just find in the textbook... You can just read 
This is an author-produced, peer-reviewed version of this article. The final, definitive version of this document can be found online at Educational Media International, published by Taylor and Francis. Copyright restrictions may apply. Doi: 10.1080/09523980902933425

the book instead. That's Force-feed type of education, not good. Would be better just to assign us some projects, let us try to figure out, and let us ask him [the teacher] if we have questions - this way is better... For example, in this project we just completed, it'd be difficult to lecture about. He [the instructor] has provided examples, then a lot more you have to do yourself. When you do it, you have to think, so you'll learn much more. Except for Excel, we've learned a lot more, because in addition to what I was in charge of, I had 2 group members, we'd discuss together when we had questions. Because not only we had to think of a good topic, but also we had to find similar things as examples, and use them as references...

Like [when we worked on] coding, we had seen other groups' posts before checking out the examples from the teacher, so we were able to figure out how it was supposed to be done... Also, when we designed the questionnaire, there was one question item, we listed quite a lot options for that one item and originally was asking [the respondent] to choose just one of them. Then a classmate from another group suggested that we should allow multiple choices, otherwise with 20 some options it'd be really difficult to choose just one. So we changed it to the way it is...

Participants also expressed very favorable views on online PBL, based on their first experience in this study: [compared to PBL] The traditional way of teaching is boring: simply do whatever the teacher does or asks you to do. In an online course like this one, you can decide what you want to read, where to go, you may also do things and practice [skills] at the same time. Compared to traditional teaching, of course online is more, lively. In traditional classrooms, you go to class, perhaps ask a question or two, if any; in online courses, the teacher may teach you some then you go do it and learn more. (QuanWen, female, superior participation)

At the same time, 16 of the 48 interviewees thought that in terms of technical skills with Excel, they might have learned the same or even more in traditional classroom lectures with drill and practice. All participants felt that they had spent more time on this 6-week project than they normally would have to in a traditional 13-week course. And thus, the demanding nature of PBL was a major concern of the participants and caused some reservations on their willingness to opt for a similar experience in the future.

Many interviewees commented that an instructor should "teach" them all the skills involved in the project, and assign the project as a drill afterwards instead of having them explore, search, learn and construct their own knowledge and skills. These students, who were used to lecturing and drill and practice, reported that they would be comfortable remaining that way. Participants complained that PBL was time consuming and frustrating, being too different from what they were used to, and the online delivery mode added even more frustration with the virtual distance between the instructor and students, and amongst learners themselves as well. They would like to have periodical meetings with the instructor as a class, to seek confirmation in person about what the instructor would expect or like to see in the final products, or simply tell them what to do, instead of having to figure out how to solve the problem and having too much control or stakes in their hands. For typical "good" students, who did well in standardized tests, PBL was not considered helpful, since typical assessments in Taiwan did not involve illstructured problem solving or the like.

Instructor's expected role in online PBL

Participants were confused and frustrated with the instructor's changed role in online PBL. They expressed that instructor's availability, accessibility, and timely help were very important to reduce anxiety and to motivate participation. Consistently, students voiced their concerns, confusions and discomfort of not having a teacher showing in person, as they then felt uncertain and "unreal" about the learning, or "being-taught" experience to be more accurate. Some participants stressed the highly desired immediate, physical availability of the instructor like the following:

I don't like online course because I can't ask the instructor questions and get an instant answer. (PeiYu, female, average participation)

If I feel the instructor is watching me I will work harder, but in an online environment I can't feel the instructor, thus sometimes I even forgot to log into the [course management] system. (JiJia, female, poor participation)

I felt empty because I cannot get in touch with the instructor and classmates. (HuiPing, (female, average participation,)

I need authority, power to force me to sit in the classroom. (JunHong, male, poor participation) 
All participants were used to seeing instructors physically in the classroom and typically were comfortable learning through lengthy lectures from the instructor; and thus they expected the same or very similar in online learning. When they did not get to meet in person regularly with the instructor, some students even forgot to logon to the course website or to read the learning materials or resources provided online. As it was their first PBL experience, many were confused about their role as active learners, information seekers and knowledge builders in the constructivist approach to learning, as well as the instructor's changed role as a facilitator, guide and moderator instead of the knowledge giver. The difficult transition was quite reasonable given the strong traditional perceptions of teaching and learning in Chinese culture, which values instructors as the authority of knowledge and the center of "teaching", and views education as mostly about "teaching" as opposed to "learning". Analysis of our online observation notes, instructor's field notes and students' online discourse showed that students constantly sought confirmation from the authority, the teacher, even when all necessary instructions and learning materials were made available online and the teacher was moderating the online activities. The students were often hesitant to move forward without checking with the instructor in private for a confirmation. They wanted some personal confirmation, not necessarily elaboration or explanation, but often a simple "yes" or "no" type of confirmation from the teacher.

The instructor in this study was closely monitoring the online collaboration process and played a role of a facilitator or moderator rather than "knowledge giver". But some students didn't understand that the teacher was trying to stimulate their own thinking, or why he would not just tell them what to do. Students cared about grades, and conventionally, doing what the teacher wanted or liked would get them a good grade. Therefore, a few interviewees disvalued the online videos and other learning materials, because "the handouts didn't give me a simple answer or solution to the problem we needed to solve. Instructor should tell us what to do and how to do it directly" (YuJie, female, poor participation). Thus they wanted the teacher to tell them, simply and directly, what to do and how to solve the problem. In addition, students were not used to the "openness" of open-ended learning like in this study.

\section{Discussion}

Consistent to research on cultural impacts on learning (Allen \& Boykin, 1992; Fwu, \& Wang, 2002; Wilson, 2001), this study has found intriguing phenomena related to the adoption of western teaching and learning methods, PBL and online collaborative learning in this case, in an oriental cultural setting, Taiwan. Clearly, online learning, collaborative learning and PBL all challenged the undergraduate students in this study in many ways. Experiencing all of them once for a first time, students obviously were facing serious challenges, including particularly the virtual distance, the changed role of an instructor, the new ways of teaching and learning, and collaborating with peers. The dramatic changes from traditional, teacher-led and teacher-centered face-to-face learning to project-based, online collaborative learning, in which students have to take more active initiatives, have led to a lot of confusions, anxiety, and even discomfort for undergraduate students in Taiwan.

Many students acknowledged appreciation for PBL as they experienced the learning benefits in this study. Many more, however, were challenged in many ways. Contradictory to the learning-from-the-master tradition (Ku et al., 2004), in PBL students were expected to take more active control over their own learning (Barron, 1998; Frank et al., 2003; Han \& Bhattacharya, 2001; Heckendorn, 2002), which many participants found overwhelming and confusing, with all the differences from what they were used to. Evidently, educators need to pay closer attention to the cultural influences on students' adoption of innovative learning methods. In similar cases, to help overcome the cultural obstacles, one may need to provide more scaffolding, modeling and modifications to sensitively adopt new methods originated in a foreign culture.

Chinese culture is very different from the western (Hofstede, 1986, 2001). And as a result, Chinese students would learn differently from those in the Western culture (Allen \& Boykin, 1992; Fwu, \& Wang, 2002; Hofstede, 1986, 2001; Wilson, 2001). For example, given the collectivist nature, Chinese students normally would try to avoid disagreeing, arguing or criticizing their peers as an effort to maintain harmony and avoid or suppress conflicts. They also have a much stronger tendency to avoid uncertainty, and thus when Chinese students are exposed to a demanding, open-ended project with the instructor serving as a facilitator rather than a knowledge giver, they feel highly uncertain and unsecured. In addition, the open-ended nature and PBL approach to the course, the lack of nonverbal cues (Chen \& Starosta, 1998; Hall, 1976) and low level of perceived social presence of the instructor (Powell \& Harville, 1990; Tu, 2001) have all together exemplified the uncertainty avoidance dimension in the cross-cultural experience (Hofstede, 2001; Vatrapu \& Suthers, 2007). Those students, who tend to avoid uncertainly would be 
more comfortable with clear structures set upfront by the instructor, and would try to seek clear and accurate instructions from the instructor, especially when assigned with ill-structured tasks like problem solving projects. And, thus, when the problem is less well defined and it is unclear how to approach the problem solving task, with step-by-step instructions from the instructor, as in a PBL experience in this study, these students may feel highly challenged both cognitively and emotionally, with confusions and strong desire for cook-book type of procedural instructions. And, when step-by-step guidance is not provided by the instructor, even with good explanations on the benefits to stimulate active knowledge seeking and construction, the students may not appreciate the extra challenges but blame the instructor for the extra work they have to do, in some case, even with satisfactory task outcomes.

Participants in this study shared an understanding that cooperation equaled collaboration. Collaboration requires a mutual engagement throughout the process, while cooperation is often a division of role and labor. Participants in this study reported that at the beginning of the team work, collaboration was more evident while at other stages cooperation was dominant. More interestingly, outside of the group students viewed the learning experience more of a competition between groups and had serious reservations for collaboration or peer modeling, which is very understandable given the long existing, highly competitive, norm-reference test system in Taiwan. Very different from the western educational system, in Taiwan, student assessments are mostly, if not all conducted in a competitive fashion, with typically everyone knowing everyone's grade. So when the share of peers projects and comments and suggestions from peers had actually helped improve the quality of the projects, those who had helped their peers to outperform themselves felt and unfair and was quite upset, thus they wouldn't be in favor for collaborations or peer reviews outside of their group, with peers who they were always competing against. Also, as the participants indicated, the dominant assessment systems in Taiwan all utilize standardized tests, and thus PBL seemed not only challenging but more importantly, irrelevant and useless, because they would not be evaluated on how well they were able to problem solve, or collaborate with peers, or complete a comprehensive project like they did in this study.

The participants' focus on competition in collaborative learning environments was consistent with previous research conducted (Nicholson, Gelpi, Young, \& Sulzby, 1998; Shaffer, 2004; Turner, Styers, \& Daggs, 1997; Yu, 1998). For example, Shaffer (2004) found that peer competitions had positive effects on learning, as students learned from modeling their peers. Many participants in this study had also recognized the benefits of learning from their fellow students as well. However, participants in this study expressed serious reservations for collaborations or peer review outside of the group. Chinese students are used to compete against their peers for greater grades in academic exams, which are the determining criterion for school admissions and school choice. How to facilitate and promote peer collaborative learning, and to help students learning from collaborative experiences, despite the highly competitive culture lays out serious challenges for educators in Taiwan and similar cultural settings.

Interestingly, in the interviews, those with lower level of participation in the study seemed to be less vocal in the focus groups. We think this is understandable, especially when those of higher participation levels, who also tended to be higher achievers obviously felt much stronger when they complained about how much their peers had learned from their projects or drafts. Again with the focus on winning in this competition, not collaboration, it is certainly understandable that those higher achievers felt much stronger regarding competition vs. collaboration, while those average or lower achievers for face-saving reasons choose not to boast about how much they might have learned from their peers. And again, we clearly sense the strong competitions among the participants. Perhaps with individual interviews, some of these lower achievers or less active participants may be more comfortable to speak out more, with less peer pressure, which might have been present in the focus groups.

The findings of this study deepen our understanding of students' perceptions of online collaboration and PBL in Taiwan and the powerful cultural influences on students learning experiences. The findings also generate special insights into the cultural roots of students' perspectives in Taiwan as well as a similar Asian culture. Such insights will help us to develop more effective instructional strategies to better implement and adopt learning methods that originated in a different culture.

Practically, the study generates strong implications on the implementation of innovative online learning in Taiwan and a similar cultural setting. For example, consistent with Murphy and Yum's (1998) suggestion, we recommend that learners of Chinese culture should be encouraged and trained to develop self reflections and self guidance in response to the less structured distance learning environments. And to help lower students' anxiety level, especially that associated with the desire for certainty and authority from the instructor, appropriate support and external 
rewarding, in addition to internal rewards gained in the learning process need to be in place, especially at the early stage of an innovative learning approach in a different culture. In the long term, the educational assessment system has to change and address higher order thinking skills, otherwise the promotion of these skills in the innovative instructional approaches will continue to be challenged and questioned, and not valued or appreciated by either the students, parents or the bigger social systems (e.g., for school admission, social recognition, or promotion, etc.).

An additional strength of the study lies in the bilingual research method. As all data were collected in Chinese with bilingual data analysis conducted in both English and Chinese and reported here in English, we were able to cross check our understandings and interpretations of the data in both languages and cultures. Although some original meaning in Chinese might have been lost or modified due to the linguistic and cultural differences in the two very different languages, as native Chinese speakers with extensive teaching and research experiences in both China and the United States, we are confident that our bilingual analysis and member check efforts have minimized such possible loss of the richness of the data, and indeed has enriched the study with cross-cultural references as we went back and forth in two languages throughout the research process. 
This is an author-produced, peer-reviewed version of this article. The final, definitive version of this document can be found online at Educational Media International, published by Taylor and Francis. Copyright restrictions may apply. Doi: 10.1080/09523980902933425

\section{References}

Allen, B., \& Boykin, W. (1992). African-American children and the educational process: Alleviating cultural discontinuity through prescriptive pedagogy. School Psychology Review, 21(4), 586-596.

Barron, B. (1998). Doing with understanding: Lessons from research on problem- and project-based learning. Journal of the Learning Sciences. 7(3\&4), 271-311.

Chard, S. C. (1992). The Project Approach: A Practical Guide for Teachers. Edmonton, Alberta: University of Alberta Printing Services.

Chen, G. M., \& Starosta, W. J. (1998). Foundations of intercultural communication, Allyn and Bacon, Boston, MA.

Crump, B.J. (2004) New Arrival students: mitigating factors on the culture of the computing learning environment. Proceedings of the sixth conference on Australian computing education, 30, 49-56.

Dillenbourg, P. (1999). What do you mean by "collaborative learning"? In P. Dillenbourg (Ed.), Collaborative learning: Cognitive and computational approaches (pp. 1-16). Amsterdam, NL: Pergamon, Elsevier Science.

Frank, M., Lavy, I. \& Elata, D. (2003). Implementing the project-based learning approach in an academic engineering course. International Journal of Technology and Design Education, 13, pp. 273-288.

Fwu, B-J. \& Wang, H-H. (2002). The Social Status of Teachers in Taiwan. Comparative Education. 38(2), 211-224.

Hall, E. T. (1976). Beyond culture, Garden City, NY: Anchor

Han, S. \& Bhattacharya, K. (2001). Constructionism, Learning by Design, and Project-based Learning. In M. Orey (Ed.), Emerging perspectives on learning, teaching, and technology.

Heckendorn, R. B. (2002). Building a Beowulf. Leveraging research and department needs for student enrichment via project based learning. Computer Science Education, 12(4), 255-273.

Hofstede, G. (1986). Cultural differences in teaching and learning. International Journal of Intercultural Relations, $10,301-320$.

Hofstede, G. (2001). Culture's consequences: Comparing values, institutions and organisations across nations (2nd ed.). Thousand Oaks, CA: Sage.

Katz, L.G. \& Chard, S.C. (2000). Engaging Children's Minds: the Project Approach. Norwood, NJ: Ablex.

Ku, H. Y., Pan, C. C., Tsai, M. H., Tao, Y., \& Cornell, R. A. (2004). The impact of instructional technology interventions on Asian pedagogy. Educational Technology Research and Development, 52(1), 88-92.

Lee, D. (2004). Web-based instruction in China: cultural and pedagogical implications and challenges. Educational Technology Research and Development, 52(1), 101-105.

Macmillan, B. (1975). Project memo 1: Project-based learning in Open University courses (Project memo series). Milton Keynes, UK: Open University, Institute of Educational Technology.

Murphy, D., \& Yum, J. C. K. (1998). Understanding Hong Kong distance learners.Distance Education, 19(1), 6480. 
Nicholson, J., Gelpi, A. Young, S., \& Sulzby, E. (1998). Influences of gender and open-ended software on first graders' collaboration composing activities on computers. Journal of Computing in Childhood Education, 9(1), 3-42.

Powell, R \& Harville, B (1990) The effects of teacher immediacy and clarity on instructional outcomes: an intercultural assessment, Communication Education, 39(4), 369-379.

Robinson, B. (1999). Asian learners, Western models: some discontinuities and issues for distance educators. In R. Carr, O. J. Jegede, T-M Wong, \& K-S Yuen (Eds.), The Asian distance learner (pp. 33-48). Hong Kong: The Open University of Hong Kong.

Roschelle, J., \& Teasley, S. (1995). The construction of shared knowledge in collaborative problem solving. In C. O'Malley (Ed.), Computer-supported collaborative learning (pp. 69-197). Berlin, Germany: Springer Verlag.

Shaffer, D. W. (2004). When computer-supported collaboration means computer-supported competition: professional mediation as a model for collaborative learning. Journal of Interactive Learning Research, 15(2), 101-115.

So, H. J., \& Brush, T. (2008). Student perceptions of collaborative learning, social presence and satisfaction in a blended learning environment: Relationships and critical factors. Computers \& Education, 51(1), 318-336.

Stake, R. E. (1995). Art of Case Study Research. Thousand Oaks, CA: Sage.

Tu, C. H. (2001). How Chinese perceive social presence: An examination of interaction in online learning environment. Education Media International, 38(2), 45-60.

Turner, J. C., Styers, K. R., \& Daggs, D. G. (1997). Encouraging mathematical thinking. Mathematics Teching in the Middle School, 3, 66-72.

Vatrapu, R. \& Suthers, D. (2007). Culture and Computers: A Review of the Concept of Culture and Implications for Intercultural Collaborative Online Learning. In T. Ishida, S. R. Fussell, \& P. T. J. M. Vossen (Eds.) Intercultural Collaboration First International Workshop, IWIC 2007 Kyoto, Japan, January 25-26, 2007 Invited and Selected Papers (pp. 260-275). Berlin, Germany: Springer Verlag.

Wang, M. (2007). Designing Online Courses that Effectively Engage Learners from Diverse Cultural Backgrounds. British Journal of Educational Technology, 38(2), 294-311.

Wilson, M. S. (2001). Cultural considerations in online instruction and learning, Distance Education, 22(1), 52-64.

Yin, R. K. \& Campbell, D. T. (2002). Case Study Research: Design and Methods, Vol. 5. Thousand Oaks, CA: Sage.

Yu, F-Y. (1998). The effects of cooperation with inter-group competition on performance and attitudes in a computer-assisted science instruction. The journal of Computers in Mathematics and Science Teaching, 17(4), 381-395.

Zhang, K., \& Hung, J. (2006). E-learning in Taiwan: Policies, practices, and problems. International Journal of Information and Communication Technology Education, 2(1), 37-52. 


\section{Appendix A: Semi-structure Interview Protocol}

I. Online Collaboration Experience:

1. Tell me about your online learning experience...how has it been, are you enjoying it?

2. Can you tell me about the teacher's involvement on the LMS? How do you like it?

3. How did you feel about the teacher's appearances on the LMS? why?

4. When you saw a message posted by the teacher, what did you do typically? Why? Examples?

II. Project-Based Learning Experience:

1. Tell me about your PBL experience... how has it been?

2. Can you tell me something about the projects your group has worked on? 